\title{
Target vessel displacement during fenestrated and branched endovascular aortic repair and its implications for the role of traditional computed tomography angiography roadmaps
}

\author{
Marloes M. Jansen ${ }^{1} \wedge$, Merel van der Stelt ${ }^{2}$, Stefan P. M. Smorenburg ${ }^{2}$, Cornelis H. Slump ${ }^{3}$, \\ Joost A. van Herwaarden ${ }^{\wedge}{ }^{\wedge}$ Constantijn E. V. B. Hazenberg ${ }^{\wedge} \wedge$ \\ ${ }^{1}$ Department of Vascular Surgery, University Medical Center Utrecht, Utrecht, the Netherlands; ${ }^{2}$ Technical Medicine, University of Twente, \\ Enschede, the Netherlands; ${ }^{3}$ Department of Robotics and Mechatronics, University of Twente, Enschede, the Netherlands
}

Contributions: (I) Conception and design: All authors; (II) Administrative support: MM Jansen, M van der Stelt, SPM Smorenburg; (III) Provision of study materials or patients: MM Jansen, M van der Stelt, SPM Smorenburg, JA van Herwaarden, CEVB Hazenberg; (IV) Collection and assembly of data: MM Jansen, M van der Stelt, SPM Smorenburg, JA van Herwaarden, CEVB Hazenberg; (V) Data analysis and interpretation: MM Jansen, M van der Stelt, SPM Smorenburg; (VI) Manuscript writing: All authors; (VII) Final approval of manuscript: All authors.

Correspondence to: Marloes M. Jansen, MSc. Universitair Medisch Centrum Utrecht, Postbus 85500, Huispostnummer G04.129, 3508GA Utrecht, the Netherlands. Email: m.m.jansen-38@umcutrecht.nl.

Background: This retrospective study quantifies target vessel displacement during fenestrated and branched endovascular aneurysm repair due to the introduction of stiff guidewires and stent graft delivery systems. The effect that intraoperative vessel displacement has on the usability of computed tomography angiography (CTA) roadmaps is also addressed.

Methods: Patients that underwent fenestrated or branched EVAR were included in this retrospective study. Two imaging datasets were collected from each patient: (I) preoperative CTA and (II) intraoperative contrastenhanced cone beam computed tomography (ceCBCT) acquired after the insertion of the stiff guidewire and stent graft delivery system. After image registration, the 3D coordinates of the ostium of the celiac artery, superior mesenteric artery, right renal artery and left renal artery were recorded in both the CTA and the ceCBCT dataset by two observers. The three-dimensional displacement of the ostia of the target vessels was calculated by subtracting the coordinates of CTA and ceCBCT from one another. Additionally, the tortuosity index and the maximum angulation of the aorta were calculated.

Results: In total 20 patients and 77 target vessels were included in this study. The ostium of the celiac, superior mesenteric, right renal and left renal artery underwent non-uniform three-dimensional displacement with mean absolute displacement of 8.2, 7.7, 8.2 and $6.2 \mathrm{~mm}$, respectively. The average displacement of all different target vessels together was $7.8 \mathrm{~mm}$. A moderate correlation between vessel displacement and the maximum angulation of the aortoiliac segment was found (Spearman's $\rho=0.45, \mathrm{P}<0.05$ ).

Conclusions: The introduction of stiff endovascular devices during fenestrated or branched EVAR causes significant, non-uniform displacement of the ostium of the visceral and renal target vessels. Consequently, preoperative CTA roadmaps based on bone registration are suboptimal to guide target vessel catheterization during these procedures.

Keywords: Fenestrated endovascular aneurysm repair (FEVAR); branched endovascular aneurysm repair (BEVAR); multimodal imaging; imaging, three-dimensional (3D); endovascular procedures

^ ORCID: Marloes M. Jansen, 0000-0001-9638-4384; Cornelis H. Slump, 0000-0001-6544-4567; Joost A. van Herwaarden, 0000-00031165-5179; Constantijn E. V. B. Hazenberg, 0000-0002-2408-2720. 
Submitted Sep 18, 2020. Accepted for publication Mar 15, 2021.

doi: 10.21037 /qims-20-1077

View this article at: http://dx.doi.org/10.21037/qims-20-1077

\section{Introduction}

Fenestrated and branched endovascular aneurysm repair (FEVAR and BEVAR) provide minimal invasive treatment to patients with complex juxta-renal and supra-renal aortic aneurysms (1). The procedure involves a main stent graft body that contains fenestrations or branches which are connected to the visceral and renal arteries with bridging stents. This method ensures that the aneurysm sack is excluded from circulation, while the blood flow towards the vital organs is preserved (2). Catheterization of these visceral

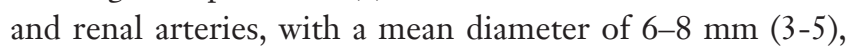
can be challenging due to the size of the aneurysm, vessel tortuosity, stenosis of the vessel origin or misalignment of the main stent body. The technical complexity of target vessel catheterization can lead to high radiation exposure, high contrast volumes and long procedure times. Optimal visualization of the target vessels and their orientation in three-dimensional (3D) space plays a key role in the catheterization process.

Image fusion is one of the techniques that enables $3 \mathrm{D}$ visualization of the target vessels during FEVAR and BEVAR (6). Image fusion allows its user to combine intraoperative fluoroscopy with patient-specific preoperative imaging, typically computed tomography angiography (CTA). The resulting image contains real-time visualisation of the endovascular devices (in 2D) in combination with a high-resolution projection of the arteries of interest (in 3D) which can be a powerful tool to improve image guidance. Throughout this paper, the term 'image fusion' will be used to refer to this imaging technique.

Image fusion relies on rigid image registration (i.e., alignment) of the preoperative CTA with the patient's ontable anatomy. This process requires an intraoperative cone beam computed tomography (CBCT) scan (3D/3D registration) or, two biplane fluoroscopy images $(2 \mathrm{D} / 3 \mathrm{D}$ registration). The main landmarks for alignment of the scans are the pelvis, vertebrae and arterial calcifications. After initial image registration, a status quo of the patient anatomy is assumed. In reality the use of endovascular devices, such as stiff guidewires and stent graft delivery systems, causes the arteries to stretch and elongate (7). This geometrical deformation leads to a mismatch between actual position of target vessels and their representation on the roadmap.

This study assesses target vessel displacement due to the introduction of a stiff guidewire and stent graft delivery system during FEVAR and BEVAR procedures. The displacement of the four target vessels in relation to each other is assessed in closer detail. Additionally, anatomical factors were identified to predict the extent of vessel displacement prior to the procedure.

\section{Methods}

\section{Patient data}

The study was conducted in accordance with the Declaration of Helsinki (as revised in 2013). The study was approved by the ethics board of the METC Utrecht (Protocol No.: 19-381/C) and individual consent for this retrospective analysis was waived. All patients that had undergone FEVAR or BEVAR within our institution between January 2015 and January 2017 were screened for this study, and their data was anonymized.

All patients had undergone preoperative CTA to assess suitability for endovascular intervention, and to design the custom-made fenestrated or branched stent graft. Additionally, all patients had received an intraprocedural contrast-enhanced CBCT (ceCBCT) as part of our institution's standard protocol for complex endovascular treatment of juxta- and suprarenal aneurysms. We excluded patients with preoperative CTA scans that deviated from our own in-house protocol for visualization of the abdominal aorta, since we wanted to assure homogenous image quality. Patients with pre-existing aortic or thoracic stent graft were also excluded from the study, as the presence of a rigid stent graft could influence vessel deformation.

\section{Imaging}

\section{CTA scans}

The preoperative CTA scan of the patient was acquired 1-7 months prior to the FEVAR or BEVAR procedure, with a Philips Brilliance ICT 256 Slice Scanner (Philips, Best, the Netherlands). To minimize motion artefacts, the scans were acquired with ECG-triggering and breath-hold after inhalation. The scan parameters were: tube voltage, 

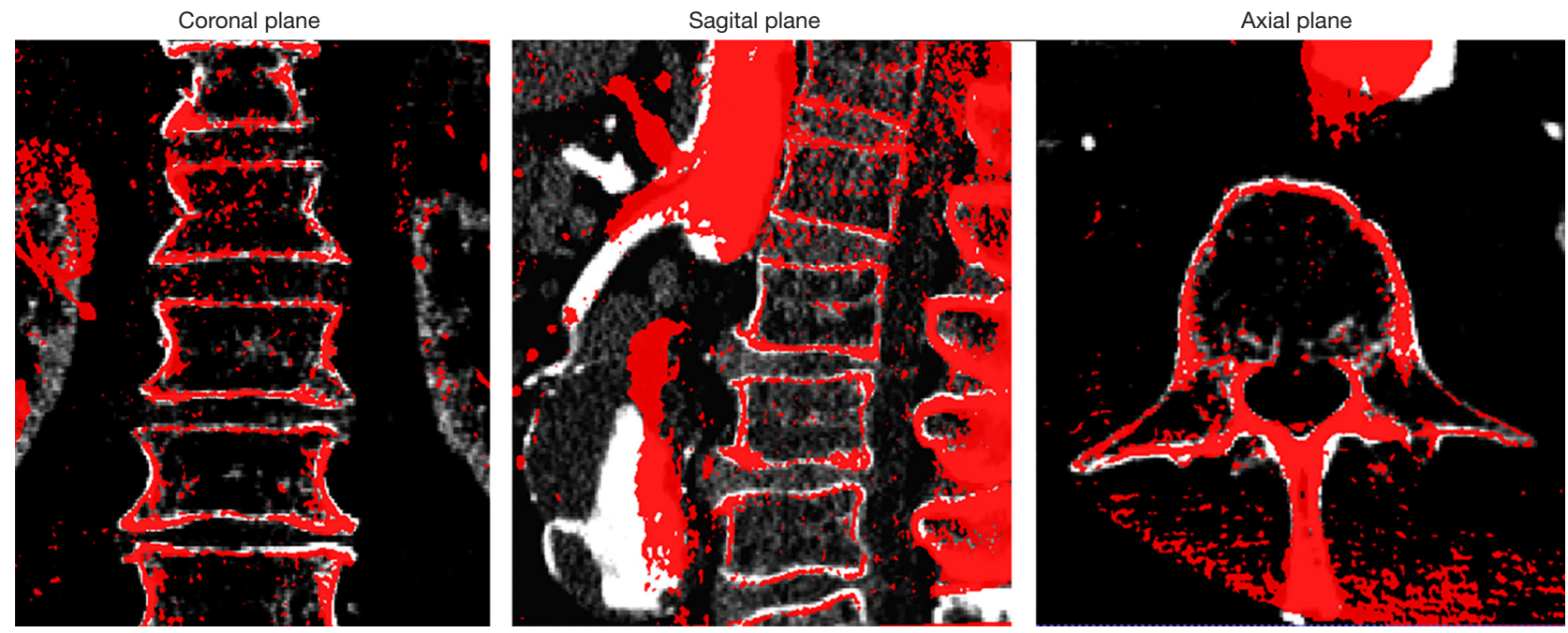

Figure 1 Image registration based on the bony structures of the vertebrae. The vertebrae of both imaging datasets were aligned using automatic scaling and manual translation and rotation. The computed tomography angiography (CTA) dataset is displayed in black-andwhite, and the contrast-enhanced cone beam computed tomography (ce-CBCT) dataset is superimposed in red including the outline of the (undeployed) stent graft. Coronal, sagittal and axial reconstructions were used to confirm successful alignment of both imaging datasets, as seen here.

100-120 kV; mAs, 183-189; reconstructed voxel size, $\leq 0.98 \mathrm{~mm} \times 0.98 \mathrm{~mm} \times 1 \mathrm{~mm}$ (range, $0.45 \mathrm{~mm} \times 0.45 \mathrm{~mm}$ $\times 1 \mathrm{~mm}-0.98 \mathrm{~mm} \times 0.98 \mathrm{~mm} \times 1 \mathrm{~mm})$. Intravenous contrast was injected with a solution of $80 \mathrm{cc}$ (patient weight $<70 \mathrm{~kg}$ ) or $100 \mathrm{cc}$ (patient weight $>70 \mathrm{~kg}$ ) of contrast fluid (Ultravist-300 mg/mL, Bayer AG, Leverkusen, Germany) and $40 \mathrm{cc}$ saline at an injection rate of $5 \mathrm{cc} / \mathrm{s}$. Scan delay was based on prior bolus triggering with a threshold level of $125 \mathrm{HU}$ at the level of the renal arteries.

\section{CeCBCT scans}

The ceCBCT scan was performed during an endovascular procedure performed in a hybrid operating room with Philips Allura FD-20 fixed fluoroscopy system (Philips, Best, the Netherlands). All patients were treated under general anaesthesia, laying in a supine position with their left arm abducted at an angle of approximately $90^{\circ}$. Both femoral arteries and, when necessary the left brachial artery, were surgically exposed to obtain vascular access. A pigtail catheter was positioned above the level of the celiac artery through one femoral artery, and the fenestrated or branched stent-graft (custom-made Zenith ${ }^{\circledR}$ stent graft, Cook Medical, Bloomington, IN, USA) was advanced over a 0.035 " stiff guidewire (Backup Meier, Marlborough, MA, USA) from the contralateral femoral artery. The Backup Meier is one of the 'stiffer' stiff guidewires with a flexural modulus of 139.6 GPA (8). Prior to stent graft deployment, the ceCBCT was acquired. To minimize motion artefacts, a respiratory arrest was induced during the scan at positive end-expiratory pressure (PEEP) of $5 \mathrm{cmH}_{2} \mathrm{O}$. Contrast injection comprised of a patient-specific volume of contrast fluid (Ultravist-300 mg/mL) depending on the patient's BMI, kidney function and aortic lumen (range, 35-50 cc). The contrast agent was diluted with saline to a total of volume of $100 \mathrm{cc}$ and injected with an injection rate of $8 \mathrm{cc} / \mathrm{s}$. A scan delay of 2 seconds was set to ensure vessel enhancement of the arteries of interest during the complete rotation. Acquisition parameters include: tube voltage; $120 \mathrm{kVp}$, rotation; $180^{\circ}$, images; scan time; $10.2 \mathrm{~s}$. Image reconstruction was performed using metal artefact reduction to reduce metal streak artefacts caused by the stent graft and its markers. Resolution was $384 \mathrm{~mm} \times 297 \mathrm{~mm}$ $\times 384 \mathrm{~mm}$ with $1 \mathrm{~mm} \times 1 \mathrm{~mm} \times 1 \mathrm{~mm}$ voxel size.

\section{Analysis of vessel displacement}

Image registration: Image registration of the CTA with the ceCBCT was based on the bone landmarks of the vertebrae in the open-source programme ITK-SNAP 3.6.0 (9) as shown in Figure 1. First, the ceCBCT dataset was scaled to match the spatial resolution of the CTA dataset, using an automated function of the software, after which rigid 

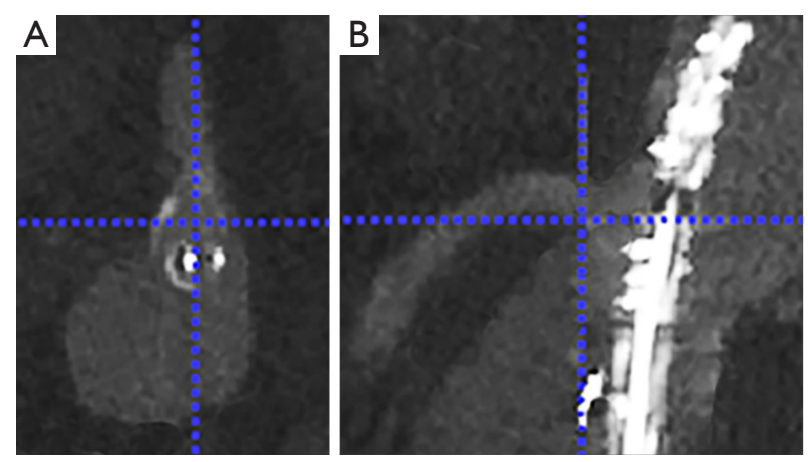

Figure 2 Placement of the landmark in the ostium of the superior mesenteric artery (SMA) in a contrast-enhanced cone beam computed tomography (ce-CBCT scan). (A) Selecting the center of the ostium of the vessel in the axial reconstruction plane; (B) selecting the distal edge of the ostium in the sagittal reconstruction plane. The undeployed stent graft is visible in both imaging planes.

transformation (rotation and translation) was used to align the ceCBCT dataset with the CTA dataset.

Correct registration of the bone landmarks is a crucial requirement for the measurement in this study. Whenever image registration failed (inability to align all vertebrae correctly) the patients were excluded from the study.

Landmarking of the ostium of the target vessels: The ostium of the celiac artery (CA), superior mesenteric artery (SMA), left renal artery (LRA) and right renal artery (RRA) were landmarked in both the CTA dataset and the ceCBCT dataset. Landmarking was performed by two researchers: one vascular surgeon who performed $>250$ FEVAR and BEVAR procedures and one experienced researcher involved in clinical imaging during $>100$ FEVAR and BEVAR procedures. The landmarks were placed in the caudal edge of the ostium of the vessel, as this edge could be distinguished consistently. This landmark was placed by first selecting the centre of the ostium of the target vessel in an axial reconstruction plane, followed by selection of the distal edge of the ostium of the vessel in the sagittal (for CA and SMA) or coronal reconstruction plane (for RRA and LRA) as shown in Figure 2. All measurements were performed using an enlarged zoom of $5 \mathrm{px} / \mathrm{mm}$ to keep measurements consistent. Each of these landmarks was recorded as a 3D coordinate. Inter observer variability was checked using the inter class correlation of both observers.

Calculation of the displacement of the ostium of the target vessels: The displacement of the ostium of the target vessels was determined by calculating the difference between the two coordinates of the CTA dataset and the
ceCBCT dataset. The displacement was calculated in the $\mathrm{x}$-, $\mathrm{y}$ - and $\mathrm{z}$ - direction, as according to Eq. [1]. The $\mathrm{x}-, \mathrm{y}$ - and $\mathrm{z}$-coordinates were defined as: $\mathrm{x}$-coordinate; left to right, $\mathrm{y}$-coordinate; anterior to posterior, $\mathrm{z}$-coordinate; caudal to cranial.

$$
\left[\begin{array}{l}
x_{d} \\
y_{d} \\
z_{d}
\end{array}\right]=\left[\begin{array}{l}
x_{C T A} \\
y_{C T A} \\
z_{C T A}
\end{array}\right]-\left[\begin{array}{l}
x_{C B C T} \\
y_{C B C T} \\
z_{C B C T}
\end{array}\right]
$$

Subsequently, the 3D displacement was calculated as the distance between the two coordinates using the law of Pythagoras, as according to Eq. [2].

$$
d=\sqrt{x_{d}{ }^{2}+y_{d}{ }^{2}+z_{d}{ }^{2}}
$$

Calculation of the uniformity of target vessel displacement in a patient: To assess the uniformity of the displacement of the renal and visceral target vessels, we calculated the intraclass correlation of the four target vessels in terms of displacement in $\mathrm{x}-, \mathrm{y}$ - and $\mathrm{z}$-axis.

\section{The effect of aortic geometry on vessel displacement}

Secondary aim of this study was to assess the influence of aortic geometry on the extent of vessel displacement. The tortuosity index and the maximum tortuosity angle were chosen for this purpose, as both required minimum measurement time during preoperative work-up.

Maximum tortuosity angle: The maximum tortuosity angle of aorto-iliac segment was measured using a $3 \mathrm{D}$ adaption of the technique described by van Keulen et al. (10), available in the 3 Mensio workstation (3Mensio Medical Imaging, Bilthoven, the Netherlands). This function measures the angulation between two line-elements of $15 \mathrm{~mm}$ along the central lumen line (Figure $3 A$ ). The highest angulation in the aortoiliac segment (from left subclavian artery until the origin of the common femoral artery) was recorded as maximum tortuosity angle.

Tortuosity index: The tortuosity indices of the aortic and iliac segment were calculated using a function available in the 3 Mensio workstation. The aortic segment ranged from the left subclavian artery until the aortic bifurcation. The iliac segment ranged from the aortic bifurcation until the origin of the common femoral artery and was measured unilaterally on the side of stent graft introduction. The tortuosity index was calculated by dividing the distance along the central lumen line $\left(\mathrm{L}_{1}\right)$ of the arterial segment, by the straight-line distance between the start and endpoint of the segment $\left(\mathrm{L}_{2}\right)(11,12)$. These measurements are shown in 

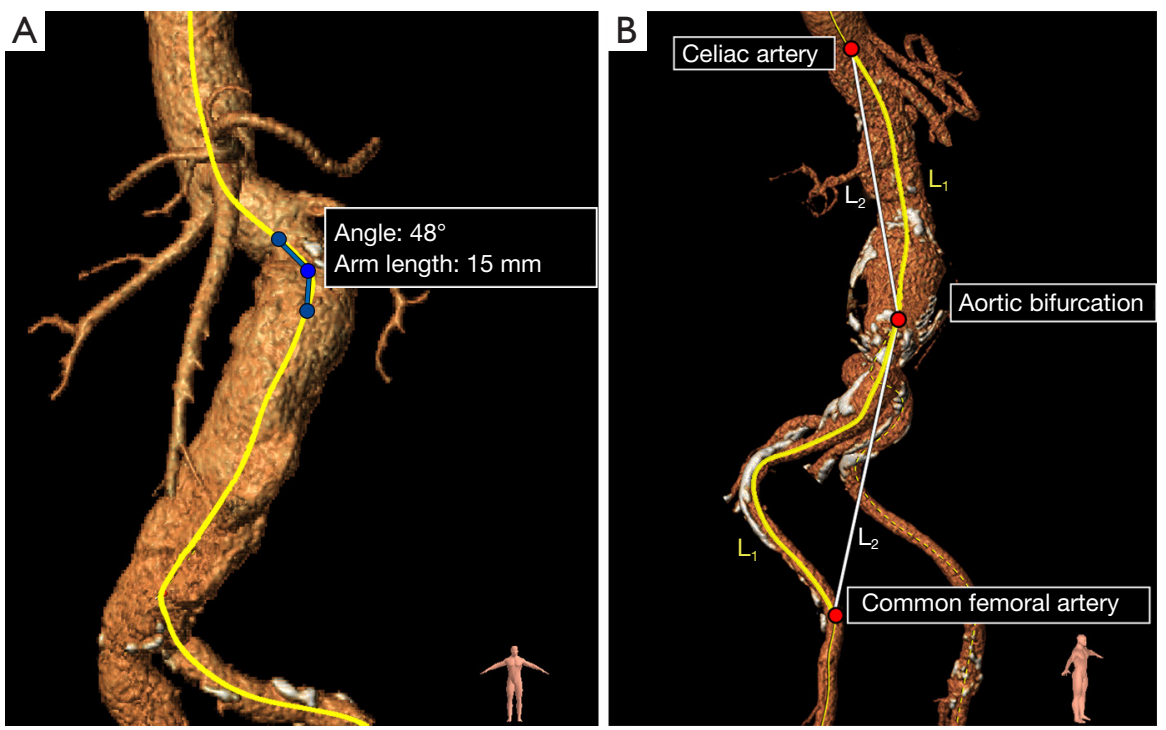

Figure 3 Calculation of tortuosity index and maximum tortuosity angle. (A) Calculation of the maximum tortuosity angle. The tortuosity angle was measured along the central lumen line (in yellow) using two $15 \mathrm{~mm}$ long line segments (in blue). The highest angulation in the aortoiliac segment was reported as the maximum tortuosity angle. (B) Calculation of the tortuosity index of the aortic and the iliac segment. The tortuosity index of the aortic segment and the iliac segment were calculated by dividing the distance along the central lumen line of the segment (L1: yellow line), by the straight-line distance between the start- and endpoint of the segment (L2: white line).

Figure 3B.

\section{Statistical analysis}

The data was analysed using $\mathrm{R}$ version 3.1.3, using the packages: ggplot, tableGrob, gtable, data.table, qqnorm, xlsx, multcomp, psych (13). Inter observer agreement was tested using the intraclass correlation (ICC), with $95 \%$ confidence interval, using a 2-way mixed effect model with absolute agreement and single measurements. Values of $<0.5$, between 0.5 and 0.75 , between 0.75 and 0.9 and $>0.9$ are indicative of poor, moderate, good and excellent reliability (14). Intraclass correlation of the target vessel displacement of the four vessels on the $\mathrm{x}^{-}, \mathrm{y}^{-}, \mathrm{z}-$ axis were also performed using ICC calculation. In which values of $<0.5$, between 0.5 and 0.75 , between 0.75 and 0.9 and $>0.9$ were indicative of poor, moderate, good and excellent correlation of the movement of all four vessels (14). Vessel displacement, tortuosity indices and maximum tortuosity angles were represented as median with range. Missing data was handled by case deletion. Correlations between the target vessel displacement and (I) the tortuosity index or (II) the maximum tortuosity angle were tested using the nonparametric Spearman's correlation test. To meet the assumption of observation independence, the independent variable comprised of the mean displacement of all four target vessels. All tests were powered to find moderate to strong linear associations only (effect size $>0.31$ ) with a power of $\geq 0.8$ and a confidence level of 0.95 .

\section{Results}

\section{Patients}

Imaging data of 74 patients were screened for eligibility, of which 43 patients were excluded due to inappropriate CTA resolution and image quality. After CTA examination another 9 patients were excluded due to pre-existing stent grafts. The remaining 22 patients were confirmed eligible for this study. Two patients were excluded during the study because of unsuccessful image registration of the vertebrae. No identifiable reason for failure of image registration could be identified, apart from differences in patient positioning. Additionally, 3 target vessels were excluded due to occlusions of the vessel origin that made it impossible to identify the distal edge of the ostia. A total of 77 target vessels from 20 patients remained available for analysis, consisting of 18 CAs, 20 SMAs, 20 RRAs, and 19 LRAs. Procedural characteristics are represented in Table 1. 


\section{Analysis of vessel displacement}

The measured displacement between preoperative CTA and intraoperative ceCBCT of the ostia of the CA, SMA, RRA and LRA are provided in Table 2. Inter observer agreement was good with an ICC of 0.89 (0.84-0.92). The median $3 \mathrm{D}$ displacement of the CA, SMA, RRA and LRA were

Table 1 Procedural characteristics

\begin{tabular}{lc}
\hline Characteristics & Number of patients [\%] \\
\hline Stent graft configuration & $2[10]$ \\
3 branches with scallop & $9[45]$ \\
3 fenestrations with scallop & $2[10]$ \\
4 branches & $4[20]$ \\
4 fenestrations & $2[10]$ \\
3 fenestrations and 1 branch & $1[5]$ \\
2 fenestrations and 2 branches & \\
Side of stent graft introduction & $17[85]$ \\
Right femoral artery &
\end{tabular}

8.2, 7.7, 8.2 and $6.2 \mathrm{~mm}$, respectively. An example of the intraoperative displacement of the arteries is visualized in Figure 4.

The displacement of the four target vessels showed a poor correlation to each other, meaning that the vessels all moved in different directions and to different extents. The intraclass correlation was $0.37(0.18-0.58), 0.16$ $(0.00-0.38)$ and $0.56(0.38-0.74)$ in terms of $x-, y-$ and $z$-axis displacement, respectively. This non-uniformity of the target vessel movement is further visualized in Figure 5.

\section{The effect of aortic geometry on vessel displacement}

Maximum tortuosity angle

The median maximum tortuosity angle of the aortoiliac segment was $58^{\circ}$ (range, $31^{\circ}-84^{\circ}$ ). A moderate monotonic relationship (Spearman's $\rho=0.45, \mathrm{P}<0.05$ ) was found between the extend of vessel displacement and the maximum tortuosity angle of the aortoiliac segment. The scatterplot is provided in Figure 6.

\section{Tortuosity index}

The median tortuosity index of the aortic and iliac segment

Table 2 Overview of absolute target vessel displacement

\begin{tabular}{|c|c|c|c|c|}
\hline Target vessel & Right-left (x-axis) & Anterior-posterior (y-axis) & Cranio-caudal (z-axis) & Total 3D vector \\
\hline Superior mesenteric artery & $4.3 \mathrm{~mm}$ (range: $0-13.7$ ) & $3.2 \mathrm{~mm}$ (range: $0-7.1$ ) & 3.0 mm (range: 0-9.0) & $7.8 \mathrm{~mm}$ (range: $2.3-14.1$ ) \\
\hline Right renal artery & 4.5 mm (range: 0-9.9) & $3.1 \mathrm{~mm}$ (range: 0-6.7) & $4.0 \mathrm{~mm}$ (range: $1.0-11.0)$ & $8.2 \mathrm{~mm}$ (range: $1.8-13.6)$ \\
\hline Left renal artery & $2.9 \mathrm{~mm}$ (range: 0-13.7) & 2.4 mm (range: 0-10.7) & 3.5 mm (range: 0-14.0) & $6.2 \mathrm{~mm}$ (range: $2.1-17.6$ ) \\
\hline
\end{tabular}

Coronal

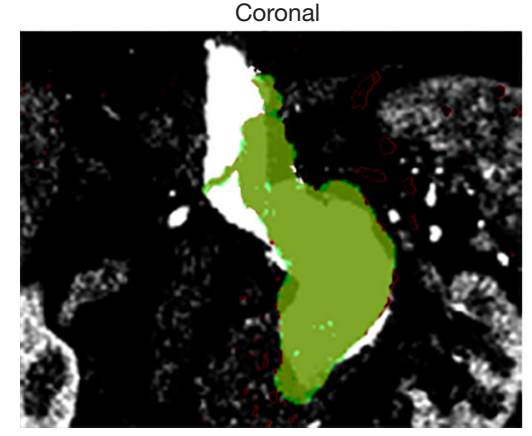

Sagittal

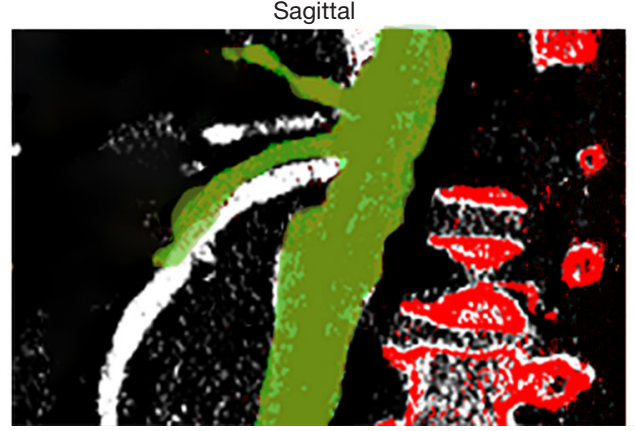

Axial

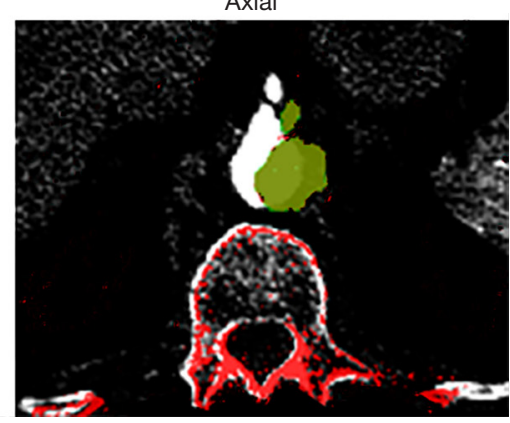

Figure 4 Target vessel displacement visualized by over-projection of the intraoperative contrast-enhanced cone beam computed tomography (ce-CBCT) on top of preoperative computed tomography angiography (CTA). The CTA scan is displayed in black-and-white and the ceCBCT is superimposed in green (arteries) and red (vertebrae and bone markers). Axial, sagittal and coronal views show the intraoperative displacement of the ostia of the target vessels. 

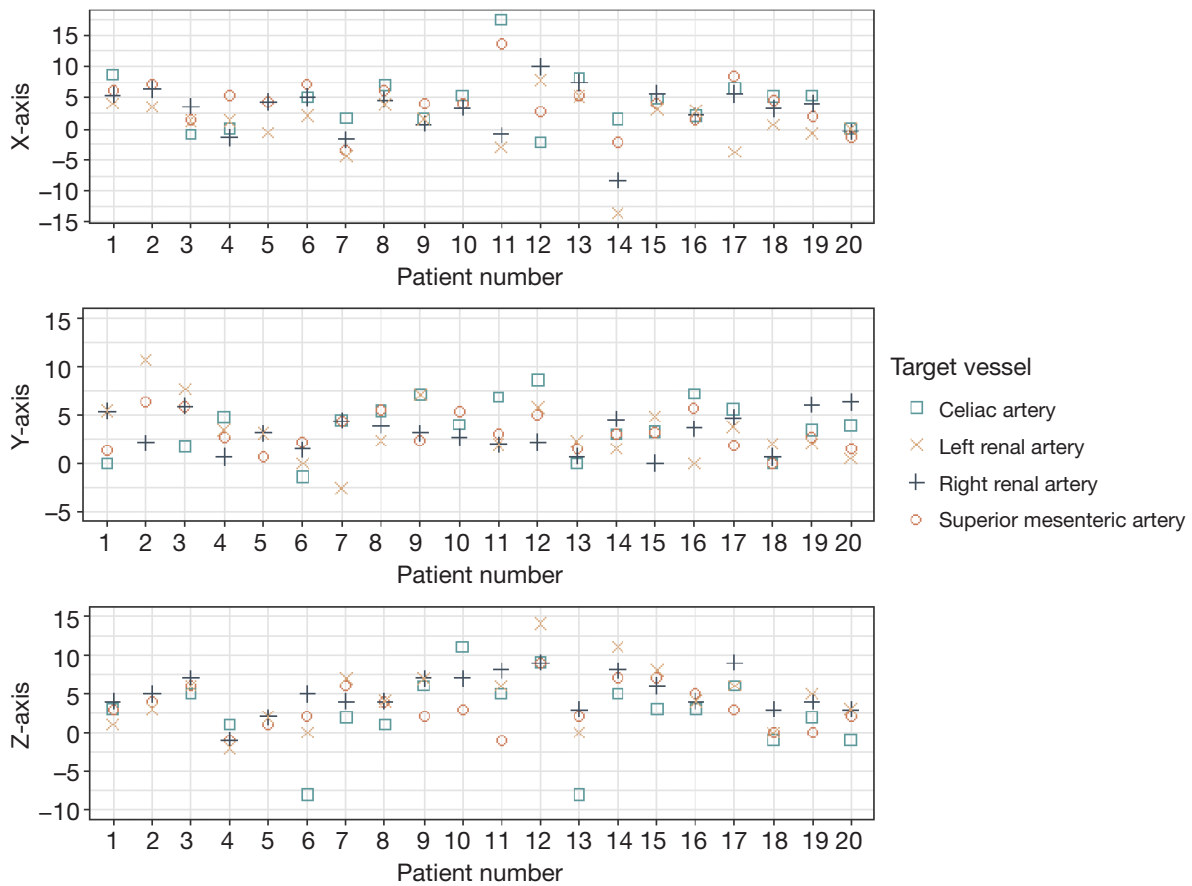

Figure 5 Non-uniform target vessel displacement. Target vessel displacement visualized in the $x^{-}, y_{-}$and $z$-axis of all twenty patients. Notice that the four target vessels in a single patient do not move as a single unit in all three axes. Instead, the displacement varies strongly amongst the different target vessels, indicating non-uniform displacement of the renal and visceral arteries. This implies that a single realignment of the roadmap to adjust for all intraoperative vascular deformation will not suffice, and that the roadmap will need to be realigned for each individual target vessel. For clarity, this scatter plot was limited to the first five patients of this study.

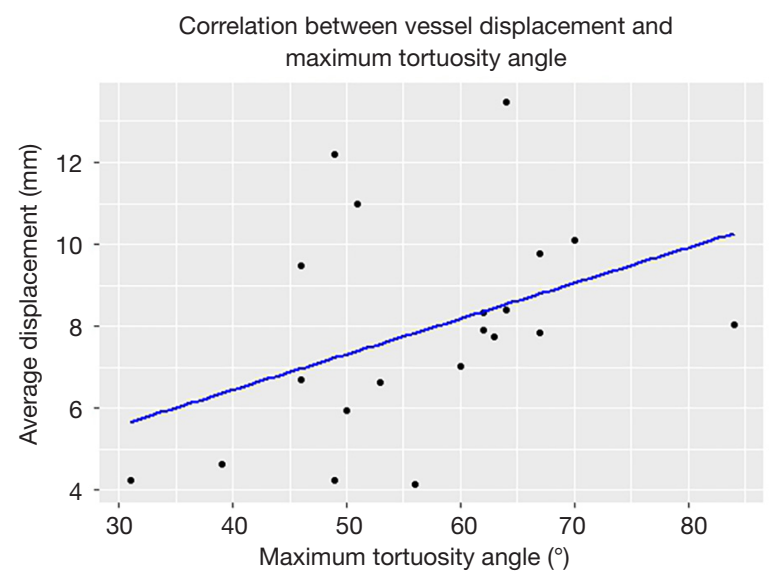

Figure 6 Association between vessel displacement and maximum tortuosity angle of the aortoiliac segment. Scatter plot of the mean vessel displacement per patient plotted against the maximum tortuosity angle of the aortoiliac segment. were 1.24 (range, 1.08-1.37) and 1.37 (range, 1.11-1.62) respectively. Based on this study, there was no significant correlation between the extend of target vessel displacement and the tortuosity index of the aortic segment $(\mathrm{P}=0.93)$, nor the iliac segment $(\mathrm{P}=0.15)$.

\section{Discussion}

This study demonstrates that the insertion of a stiff guidewire and stent graft delivery system during FEVAR or BEVAR causes significant displacement of the ostia of the visceral and renal target vessels. Selective realignment of the roadmap using an angiogram can help reduce the initial mismatch, but due to the poor correlation of the displacement across the four target vessels, the individual vessels will move differently which makes it impossible to align all target vessels at once. 
Image fusion guidance is a topic of broad interest in endovascular surgery, and its feasibility has been evaluated in a variety of procedures. Two meta-analyses have shown that the use of image fusion results in a significant reduction in radiation dose (15) and contrast material (16) during FEVAR and BEVAR procedures. However, both metaanalyses report high heterogeneity in procedural outcomes, both amongst the included studies and within these studies themselves. We postulate that the variation within the studies themselves could be related to the strong diversity in roadmap accuracy. An accurate image fusion roadmap provides guidance during target vessel catheterization and thereby reduces contrast, radiation dose and procedure time, but these procedural effects degrade quickly when the roadmap needs to be realigned repeatedly using angiography. In turn, the roadmap accuracy is affected by the extent of intraoperative vessel displacement, which differs strongly per patient: in this study, the displacement of the target vessel ostia ranged from 1.8 to $19.6 \mathrm{~mm}$. Prediction of the extent of intraoperative vessel displacement prior to the procedure, could aid patient selection for the use of image fusion. According to our findings, the maximum tortuosity angle seems to be related to the extent of target vessel displacement. However, we were unable to establish a clear cut-off point, thus making the predictive value of this measure limited.

Surgeons could consider alternative imaging methods to guide target vessel catheterization during FEVAR and BEVAR. The alternative we use in our institution, is a roadmap based on a segmented ceCBCT rather than the conventional roadmaps based on pre-operative CTA, to guide target vessel catheterization in FEVAR and BEVAR. In fact, all patients in this study were treated using the ceCBCT roadmap, rather than an actual CTA-roadmap. When acquired after the insertion of the stent graft delivery system, the ceCBCT takes into account the majority of the intraoperative deformation of the arteries. As a result, the ceCBCT roadmap accurately visualizes (I) the $3 \mathrm{D}$ position of all four ostia of the visceral and renal target vessels and (II) the run-off of these target vessels and their branches to allow stable placement of the guidewire in the distal artery. In our experience ( $>150$ procedures), these ceCBCT roadmaps seem to be more accurate than conventional CTA roadmaps. The main drawbacks of ceCBCT roadmaps are their limited field of view, the need for an additional injection of 35-50 cc contrast, and the need for intraoperative segmentation of the arteries of interest to prevent overprojection of bone and soft tissue on the roadmap. An example protocol for acquisition of ceCBCT is provided by Törnquist et al. (17).

Another fast-developing strategy to resolve the mismatch between the real-time anatomy and the image fusion roadmap, is the use of deformable (dynamic) image fusion applications (18-21). These applications generate a deformable model of the vascular geometry, in which the distance between certain subsections can be modified or stretched to allow dynamic corrections to match with the real-time anatomy. These modifications can be initiated manually, or with computerized algorithms that detect and predict the interactions between stiff guidewires and vessel structures based on finite-element simulations of the interaction between stiff guidewires and vessel structures (18-21). Validation studies of these technologies with preclinical data seems promising, but clinical prospective studies are not yet available.

Several studies have previously assessed vessel displacement due to the insertion of stiff endovascular devices using 2D angiography, but these measurements hold limited validity in the three dimensional domain $(7,22,23)$. To date, only one other study reported vessel displacement in 3D. This study by Maurel et al. (24), used similar methods to assess the $3 \mathrm{D}$ vessel displacement of the SMA, RRA and LRA during EVAR ( $\mathrm{n}=13)$ and FEVAR $(\mathrm{n}=7)$ resulting in a median vessel displacement of $6.7,6.2$ and $6.4 \mathrm{~mm}$ for the SMA, RRA and LRA, respectively. In the current study, slightly higher target vessel displacements of these target vessels were observed, which may be caused by the more proximal position of the stiff delivery system in the aorta (near the target vessels) during FEVAR and BEVAR when compared to EVAR procedures.

The present study is in accordance with the work undertaken by Kauffmann et al. (7) who previously reported non-uniformity displacement of the left and right renal artery during EVAR. A persisting mismatch of one renal artery remained visible on the image fusion roadmap after previously having aligned the contralateral renal artery.

The present study has some limitations. Firstly, the introduction of stiff endovascular devices influences the geometry of the complete vascular tree. In this study, however, we limited our analysis to the displacement of structures that are of critical importance during target vessel catheterization in FEVAR and BEVAR; the ostia of the CA, SMA, RRA and LRA.

Secondly, this study measures the displacement of the vessel ostia immediately after bone-based image registration. In clinical practice, most centres would 
perform additional adjustments based on a selective angiogram or arterial calcifications, prior to utilization of the roadmap. These adjustments are likely to reduce the overall mismatch between the image fusion roadmap and the on-table anatomy. As we aim for a robust and reproducible initial registration, we chose not to include arterial calcifications as registration markers in this study. Registration based on combination of bone markers and calcifications requires compromise and thus may have more than one possible solution. Besides, as the ostia of the different target vessels each move in different directions, realignment based on repeated angiography might be needed when alternating between target vessels. Relying on these (selective) angiograms can be disadvantageous in the unfortunate case that a main stent graft blocks the flow to one of the target vessels after deployment. In which case it is left up to estimated guessing to find the target vessel and reposition the main stent graft.

Thirdly, none of the patients in this study actually received treatment based on guidance of a CTA-roadmap, as our institution uses a different approach using an intraoperative ceCBCT a roadmap. The routine acquisition of these intraoperative ceCBCT scans, formed the basis of this unique dataset, in which the position of target vessels after the insertion of the main stent graft could be selected. In combination with a preoperative CTA this forms the opportunity to measure the intraoperative displacement between the preoperative setting and the intraoperative setting. While this study shows implications for the use of preoperative CTA roadmaps, it is important to note that the procedures themselves involved a different navigation strategy using only this intraoperative ceCBCT as a roadmap. We were therefore unable to report the effect of roadmap accuracy on procedural outcomes. Lastly, although the rigid $3 \mathrm{D} / 3 \mathrm{D}$ registration of the spine was excellent in 20 patients, alignment of the bones does not guarantee alignment of the soft tissues. Differences in respiratory phase and patient position could result in displacement of the aorta and its side branches. Pathologic disease progression between the time of CTA acquisition and intervention may also alter the vascular anatomy. Note, that during clinical use of image fusion roadmaps, these same issues would occur during image registration. In fact, discrepancies in respiratory phase are technically unavoidable when performing image fusion roadmaps in procedures performed under general anaesthesia. Preoperative CTAs/MRAs are generally acquired during self-controlled breath-hold (negative pressure ventilation), whilst intraoperative images are frequently acquired using a respiratory arrest of the mechanical ventilation (positive pressure ventilation) at positive end-expiratory pressure (PEEP). The breathing mechanisms of positive and negative pressure ventilation are different and therefore their respiratory phases are non-comparable.

According to Sailer et al. (25) the respiration induced displacement of the visceral and renal target vessels during a normal, self-controlled breathing cycle is on average $1.4 \mathrm{~mm}$, oriented in exclusively the cranio-caudal direction (z-axis). Thus, while our study outcomes may have been affected by respiratory movements, but the contribution will be small and only oriented in the cranio-caudal direction.

\section{Conclusions}

The introduction of stiff endovascular devices during FEVAR and BEVAR causes significant displacement of the ostia of the CA, SMA, RRA and LRA. The displacement of the individual target vessels within a patient shows a poor correlation to each other. Consequently, the use of preoperative CTA roadmaps will require repeated realignment when alternating between target vessels or projection angles and therefore provide suboptimal guidance for target vessel catheterization during FEVAR and BEVAR procedures.

\section{Acknowledgments}

Funding: None.

\section{Footnote}

Conflicts of Interest: All authors have completed the ICMJE uniform disclosure form (available at http://dx.doi. org/10.21037/qims-20-1077). JAvH and CEVBH have been consultants for Cook Medical, Gore Medical, Medtronic and Terumo Aortic, and have a research and consultancy agreement with Philips Medical. The other authors have no conflicts of interest to declare.

Ethical Statement: The authors are accountable for all aspects of the work in ensuring that questions related to the accuracy or integrity of any part of the work are appropriately investigated and resolved. The study was conducted in accordance with the Declaration of Helsinki (as revised in 2013). The study was approved by the ethics board of the METC Utrecht (Protocol No.: 19-381/C) and 
individual consent for this retrospective analysis was waived.

Open Access Statement: This is an Open Access article distributed in accordance with the Creative Commons Attribution-NonCommercial-NoDerivs 4.0 International License (CC BY-NC-ND 4.0), which permits the noncommercial replication and distribution of the article with the strict proviso that no changes or edits are made and the original work is properly cited (including links to both the formal publication through the relevant DOI and the license). See: https://creativecommons.org/licenses/by-nc-nd/4.0/.

\section{References}

1. Anderson JL, Adam DJ, Berce M, Hartley DE. Repair of thoracoabdominal aortic aneurysms with fenestrated and branched endovascular stent grafts. J Vasc Surg 2005;42:600-7.

2. Verhoeven ELG, Katsargyris A, Fernandes E Fernandes R, Bracale UM, Houthoofd S, Maleux G. Practical points of attention beyond instructions for use with the Zenith fenestrated stent graft. J Vasc Surg 2014;60:246-52.

3. Mohiuddin M, Mansoor A, Ali M, Hassan N. Analysis of renal artery morphometery in adults: A study conducted by using multidetector computed tomography angiography. Pak J Med Sci 2017;33:943-7.

4. Majos M, Stefa czyk L, Szemraj-Rogucka Z, Elgalal M, De Caro R, Macchi V, Polguj M. Does the type of renal artery anatomic variant determine the diameter of the main vessel supplying a kidney? A study based on CT data with a particular focus on the presence of multiple renal arteries. Surg Radiol Anat 2018;40:381-8.

5. Pinal-Garcia DF, Nuno-Guzman CM, Gonzalez-Gonzalez ME, Ibarra-Hurtado TR. The Celiac Trunk and Its Anatomical Variations: A Cadaveric Study. J Clin Med Res 2018;10:321-9.

6. Jones DW, Stangenberg L, Swerdlow NJ, Alef M, Lo R, Shuja F, Schermerhorn ML. Image Fusion and 3-Dimensional Roadmapping in Endovascular Surgery. Ann Vasc Surg 2018;52:302-11.

7. Kauffmann C, Douane F, Therasse E, Lessard S, Elkouri S, Gilbert P, Beaudoin N, Pfister M, Blair JF, Soulez G. Source of errors and accuracy of a two-dimensional/threedimensional fusion road map for endovascular aneurysm repair of abdominal aortic aneurysm. J Vasc Interv Radiol 2015;26:544-51.

8. Harrison GJ, How TV, Vallabhaneni SR, Brennan JA, Fisher RK, Naik JB, McWilliams RG. Guidewire stiffness:
What's in a name? J Endovasc Ther 2011;18:797-801.

9. Yushkevich PA, Piven J, Hazlett HC, Smith RG, Ho S, Gee JC, Gerig G. User-guided 3D active contour segmentation of anatomical structures: significantly improved efficiency and reliability. Neuroimage 2006;31:1116-28.

10. van Keulen JW, Moll FL, Tolenaar JL, Verhagen HJM, van Herwaarden JA. Validation of a new standardized method to measure proximal aneurysm neck angulation. J Vasc Surg 2010;51:821-8.

11. Chaikof EL, Fillinger MF, Matsumura JS, Rutherford RB, White GH, Blankensteijn JD, Bernhard VM, Harris PL, Kent KC, May J, Veith FJ, Zarins CK. Identifying and grading factors that modify the outcome of endovascular aortic aneurysm repair. J Vasc Surg 2002;35:1061-6.

12. Chaikof EL, Blankensteijn JD, Harris PL, White GH, Zarins CK, Bernhard VM, Matsumura JS, May J, Veith FJ, Fillinger MF, Rutherford RB, Kent KC; Ad Hoc Committee for Standardized Reporting Practices in Vascular Surgery of The Society for Vascular Surgery/ American Association for Vascular Surgery. Reporting standards for endovascular aortic aneurysm repair. J Vasc Surg 2002;35:1048-60.

13. R Core Team. R: A language and environment for statistical computing. Vienna, Austria; 2014.

14. Koo TK, Li MY. A Guideline of Selecting and Reporting Intraclass Correlation Coefficients for Reliability Research. J Chiropr Med 2016;15:155-63.

15. de Ruiter QM, Reitsma JB, Moll FL, Herwaarden JA Van. Meta-analysis of Cumulative Radiation Duration and Dose During EVAR Using Mobile , Fixed, or Fixed / 3D Fusion C-Arms. J Endovasc Ther 2016;23:944-56.

16. Goudeketting SR, Heinen SGH, Ünlü Ç, van den Heuvel DAF, de Vries JPM, van Strijen MJ, Sailer AM. Pros and Cons of 3D Image Fusion in Endovascular Aortic Repair: A Systematic Review and Meta-analysis. J Endovasc Ther 2017;24:595-603.

17. Törnqvist $\mathrm{P}$, Dias N, Sonesson B, Kristmundsson $\mathrm{T}$, Resch T. Intra-operative cone beam computed tomography can help avoid reinterventions and reduce CT follow up after infrarenal EVAR. Eur J Vasc Endovasc Surg 2015;49:390-5.

18. Gindre J, Bel-Brunon A, Rochette M, Lucas A, Kaladji A, Haigron P, Combescure A. Patient-Specific Finite-Element Simulation of the Insertion of Guidewire During an EVAR Procedure: Guidewire Position Prediction Validation on 28 Cases. IEEE Trans Biomed Eng 2017;64:1057-66.

19. Kaladji A, Dumenil A, Castro M, Cardon A, Becquemin JP, Bou-Saïd B, Lucas A, Haigron P. Prediction of 
deformations during endovascular aortic aneurysm repair using finite element simulation. Comput Med Imaging Graph 2013;37:142-9.

20. Roy D, Holzapfel GA, Kauffmann C, Soulez G. Finite element analysis of abdominal aortic aneurysms: Geometrical and structural reconstruction with application of an anisotropic material model. IMA J Appl Math (Institute Math Its Appl 2014;79:1011-26.

21. Mouktadiri G, Bou-Saïd B, Walter-Le-Berre H. Aortic endovascular repair modeling using the finite element method. J Biomed Sci Eng 2013;06:917-27.

22. Fukuda T, Matsuda H, Doi S, Sugiyama M, Morita Y, Yamada M, Yokoyama H, Minatoya K, Kobayashi J, Naito H. Evaluation of Automated 2D-3D Image Overlay System Utilizing Subtraction of Bone Marrow Image

Cite this article as: Jansen $M M$, van der Stelt $M$, Smorenburg SPM, Slump CH, van Herwaarden JA, Hazenberg CEVB. Target vessel displacement during fenestrated and branched endovascular aortic repair and its implications for the role of traditional computed tomography angiography roadmaps. Quant Imaging Med Surg 2021;11(9):3945-3955. doi: 10.21037/qims-20-1077 for EVAR: Feasibility Study. Eur J Vasc Endovasc Surg 2013;46:75-81.

23. Ahmad W, Obeidi Y, Majd P, Brunkwall JS. The 2D-3D Registration Method in Image Fusion Is Accurate and Helps to Reduce the Used Contrast Medium, Radiation, and Procedural Time in Standard EVAR Procedures. Ann Vasc Surg 2018;51:177-86.

24. Maurel B, Hertault A, Gonzalez TM, Sobocinski J, Roux M Le, Delaplace J. Evaluation of Visceral Artery Displacement by Endograft Delivery System Insertion. J Endovasc Ther 2014;21:339-47.

25. Sailer AM, Wagemans BA, Das M, de Haan MW, Nelemans PJ, Wildberger JE, Schurink GW. Quantification of Respiratory Movement of the Aorta and Side Branches. J Endovasc Ther 2015;22:905-11. 